\title{
Prostate stem cell antigen mRNA in blood is a survival after radical prostatectomy in patients with high-risk prostate cancer
}

\author{
Yoon Seok Suh ${ }^{1, *}$, Jae Young Joung ${ }^{1, *}$, Sung Han $\mathrm{Kim}^{1}$, Jeong Eun Kim ${ }^{1}$, Moon \\ Kyung Choi ${ }^{2}$, Weon Seo Park ${ }^{2}$, Sang-Jin Lee ${ }^{3}$, Ho Kyung Seo ${ }^{1}$, Jinsoo Chung ${ }^{1}$ and \\ Kang Hyun Lee ${ }^{1}$ \\ ${ }^{1}$ Department of Urology, Center for Prostate Cancer, Hospital, National Cancer Center, Goyang, Gyeonggi-do, Korea \\ ${ }^{2}$ Department of Pathology, Center for Prostate Cancer, Hospital, National Cancer Center, Goyang, Gyeonggi-do, Korea \\ ${ }^{3}$ Immunotherapeutics Branch, Research Institute, National Cancer Center, Goyang, Gyeonggi-do, Korea \\ *These authors contributed equally to this work
}

Correspondence to: Kang Hyun Lee, email: uroonco@ncc.re.kr

Keywords: prostate stem cell antigen; prostate cancer; biochemical recurrence; survival

Received: September 04, $2017 \quad$ Accepted: March 13, $2018 \quad$ Published: May 29, 2018

Copyright: Suh et al. This is an open-access article distributed under the terms of the Creative Commons Attribution License 3.0 (CC BY 3.0), which permits unrestricted use, distribution, and reproduction in any medium, provided the original author and source are credited.

\section{ABSTRACT}

Objectives: To investigate whether the preoperative detection of prostate stem cell antigen (PSCA) mRNA in blood has predictive value for biochemical recurrence, overall survival, and cancer-specific survival after radical prostatectomy in patients with high-risk prostate cancer.

Results: Median age was 67 years (interquartile range: $63-71$ ), and median follow-up was 41 months (interquartile range: 25-65). PSCA mRNA was detected in 151 patients $(51.1 \%)$. Biochemical recurrence was developed in 101 patients (34.2\%), and all-cause mortality and prostate cancer-specific mortality occurred in $17(5.7 \%)$ and $8(2.7 \%)$ patients, respectively. Kaplan-Meier analysis revealed significant differences in biochemical recurrence, overall survival, and cancer-specific survival according to PSCA mRNA positivity. Cox regression hazards model analysis showed that PSCA mRNA positivity was an independent predictor of biochemical recurrence, overall survival, and cancer-specific survival.

Conclusions: PSCA mRNA in the peripheral blood was related to poor prognosis. Detection of PSCA mRNA by polymerase chain reaction in peripheral blood can be used to predict survival after radical prostatectomy in patients with high-risk prostate cancer. Future study with larger cohort and long-term follow-up is required to confirm this finding.

Materials and methods: A total of 295 patients with high-risk prostate cancer scheduled to undergo radical prostatectomy were prospectively enrolled from 2008 to 2016. Nested reverse transcription polymerase chain reaction was used to detect cells with PSCA mRNA in peripheral blood. The predicting ability of PSCA mRNA positivity for biochemical recurrence, overall survival, and cancer-specific survival after radical prostatectomy was evaluated.

\section{INTRODUCTION}

Prostate cancer $(\mathrm{PC})$ is the second most common cancer in men and the fifth leading cause of cancer-related death worldwide [1]. In the United States of America, PC is the second leading cause of cancer-specific deaths in men, and approximately 26,120 PC-related deaths occurred in 2016 [2]. In clinically localized PC, patients harboring 
high-risk disease exhibit adverse treatment outcomes [3]. Accordingly, this subset of patients is exposed to a greater risk of biochemical recurrence (BCR) or micrometastasis after radical prostatectomy (RP) compared with patients with low- or intermediate-risk disease.

The diverse management strategies for high-risk PC yield inconsistent results, representing a challenging issue in the treatment of PC. Multidisciplinary and multimodal therapeutic approaches have been applied for the management of patients with high-risk PC [4]. Of these approaches, the combination of long-term hormone therapy and external-beam radiation therapy has been accepted as a standard care for patients with high-risk PC [5-7]. In contrast, some patients with organ-confined high-risk PC remain progression-free after undergoing $\mathrm{RP}$ alone $[8,9]$. Thus, in the era of precision medicine, selecting patients who will benefit most from RP in high-risk PC is essential.

The prostate stem cell antigen (PSCA) gene is located on chromosome 8q24.2, distal to c-Myc oncogene, and its amplification reported in a significant number of primary and metastatic PCs [10]. In our previous study [11], we found that PSCA mRNA in peripheral blood may be a good predictor of BCR in high-risk PC. In an analysis of 103 patients with high-risk disease, $P S C A$ positivity in nested reverse transcription polymerase chain reaction (RT-PCR) was found to be an independent risk factor of BCR. However, sample size was limited, and the median follow-up duration was 23 months, which may have been too short for evaluation of delayed BCR development or mortality.

Accordingly, in the present study, we investigated whether the detection of PSCA mRNA in the blood prior to operation may have predictive value for BCR, overall survival (OS), and cancer-specific survival (CSS) after RP with in patients with high-risk PC in a long-term followup study.

\section{RESULTS}

\section{Clinicopathological characteristics and PSCA detection by RT-PCR}

In all patients, the median age was 67 years (interquartile range [IQR]: 63-71), and median follow-up duration was 41 months (IQR: 25-65). The clinicopathological characteristics are summarized in Table 1. One hundred and fifty one patients (51.1\%) showed the presence of PSCA mRNA by RT-PCR. Overall, $30.5 \%$ of patients had prostate-specific antigen (PSA) over $20.0 \mathrm{ng} / \mathrm{mL}, 14.6 \%$ of patients had a biopsy Gleason score (GS) of 8-10, and $76.3 \%$ of patients had clinical stage of greater than or equal to T2c. There were no significant differences in PSA, biopsy GS, clinical stage, GS with RP specimens, extracapsular extension (ECE), seminal vesicle invasion (SVI), and surgical margin status between the two groups $(p>0.05)$.

\section{BCR following RP}

BCR was developed in 101 patients (34.2\%), and the median time to BCR was 7 months (IQR: 4-15). BCR was developed in 59 patients $(58.4 \%)$ with PSCA $(+)$ and in 42 patients $(41.6 \%)$ with PSCA $(-)$. Kaplan-Meier analysis revealed a significant difference between PSCA $(-)$ and PSCA $(+)$ groups in BCR-free survival (Logrank test, $p=0.010$; Figure 1). PSCA mRNA positivity by RT-PCR was an independent predictor of BCR (HR: 1.817, 95\% CI: 1.504-3.192, $p<0.001$; Table 2) in Cox regression hazard analysis. PSA of greater than or equal to 20 , biopsy GS of greater than or equal to 8 , and pathologic stage of greater than or equal to T3 were independent predictors of BCR as well.

\section{OS and CSS following RP}

All-cause mortality and PC-specific mortality occurred in $17(5.7 \%)$ and $8(2.7 \%)$ patients, respectively. Median time to all-cause mortality was 37 months (IQR: 29-49), and median time to PC-specific mortality was 36 months (IQR: 28-48). All-cause mortality occurred in eight with PSCA $(+)$ patients $(5.3 \%)$ and nine with PSCA $(-)$ patients $(6.2 \%)$. PC-specific mortality was developed in five PSCA $(+)$ patients $(3.3 \%)$ and three PSCA (-) patients $(2.1 \%)$. Significant differences were found in OS and CSS with respect to RT-PCR PSCA positivity after RP in Kaplan-Meier analysis (Log-rank test, $p=0.004$ and $p=0.014$, respectively; Figure 2). Cox regression hazard analysis (Table 3 ) showed that $P S C A$ mRNA positivity by RT-PCR was an independent predictor of OS (HR: 5.172, 95\% CI: 1.692-15.062, $p=0.005)$ and CSS (HR: 12.784; 95\% CI: 1.799-96.582; $p=0.008)$. Age was independent predictors of OS, and biopsy GS of greater than or equal to 8 was also independent predictors of OS and CSS.

\section{DISCUSSION}

In this study, we evaluated whether the pre-operative detection of PSCA mRNA in blood had predictive value for BCR, OS, and CSS after RP in patients with high-risk PC. PSCA mRNA in blood was related to poor prognosis with regard to $\mathrm{BCR}$, OS, and CSS.

Patients having high-risk PC tend to exhibit negative clinicopathological features and BCR compared with patients have low-risk disease. In previous studies, significantly higher risk of progression and PC-specific mortality was observed $[12,13]$. However, although radiation therapy is recommended over RP as first-line treatment in several guidelines, there is still no evidence that RP is inferior [8]. Yossepowitch et al. reported that $22-63 \%$ of PC is localized high-risk disease, and $41-74 \%$ of patients remain progression-free 10 years after treatment with RP alone [12]. Thus, selecting 
Table 1: Clinicopathological characteristics of patients according to PSCA detection by RT-PCR

\begin{tabular}{|c|c|c|c|c|}
\hline \multirow[b]{2}{*}{ Variables } & \multicolumn{3}{|c|}{ Number of patients $(\%)$} & \multirow[b]{2}{*}{$p$ value } \\
\hline & Total, $n=295$ & $\begin{array}{c}\text { RT-PCR PSCA (-), } \\
n=144\end{array}$ & $\begin{array}{c}\text { RT-PCR PSCA }(+), \\
n=151\end{array}$ & \\
\hline Age, years, median (IQR) & $67(63-71)$ & $67(62-70)$ & $67(63-71)$ & 0.457 \\
\hline PSA (ng/mL) & & & & 0.802 \\
\hline$<20.0$ & $205(69.5)$ & $99(68.8)$ & $106(70.2)$ & \\
\hline$\geq 20.0$ & $90(30.5)$ & $45(31.2)$ & $45(29.8)$ & \\
\hline GS (biopsy) & & & & 0.997 \\
\hline$\leq 7$ & $252(85.4)$ & $123(85.5)$ & $129(85.4)$ & \\
\hline$\geq 8$ & $43(14.6)$ & $21(14.5)$ & $22(14.6)$ & \\
\hline Clinical stage & & & & 0.586 \\
\hline$\leq \mathrm{cT} 2 \mathrm{~b}$ & $70(23.7)$ & $32(22.2)$ & $38(25.2)$ & \\
\hline$\geq \mathrm{cT} 2 \mathrm{c}$ & $225(76.3)$ & $112(77.8)$ & $113(74.8)$ & \\
\hline GS (RP specimen) & & & & 0.150 \\
\hline$\leq 7$ & $216(73.2)$ & $111(77.1)$ & $105(69.5)$ & \\
\hline$\geq 8$ & $79(26.8)$ & $33(22.9)$ & $46(30.5)$ & \\
\hline $\mathrm{ECE}$ & & & & 0.131 \\
\hline Positive & $153(51.9)$ & $68(47.2)$ & $85(56.3)$ & \\
\hline Negative & $142(48.1)$ & $76(52.8)$ & $66(43.7)$ & \\
\hline SVI & & & & 0.080 \\
\hline Positive & $73(24.7)$ & $29(20.1)$ & $44(29.1)$ & \\
\hline Negative & $222(75.3)$ & 115 (79.9) & $107(70.9)$ & \\
\hline Surgical margin & & & & 0.375 \\
\hline Positive & $87(29.5)$ & $46(31.9)$ & $41(27.2)$ & \\
\hline Negative & $208(70.5)$ & $98(68.1)$ & $110(72.8)$ & \\
\hline
\end{tabular}

Abbreviations: PSCA, prostate stem cell antigen; RT-PCR, reverse transcription polymerase chain reaction; IQR, interquartile range; PSA, prostate-specific antigen; GS, Gleason score; ECE, extracapsular extension; SVI, seminal vesicle invasion.

optimal treatment for these patients is essential for achieving optimal therapeutic effects, and this issue has become increasingly important since the emergence of precision medicine. However, there are no wellestablished markers for predicting prognosis after initiation of therapy, including surgery, in patients with high-risk PC. Accordingly, new markers that can predict treatment outcomes with accuracy are required.

In previous reports, inconsistent results of PSCA mRNA detection by RT-PCR with regard to its value as a predictor of treatment outcomes have been reported [14-16]. However, analysis of these studies included heterogeneous cohorts that were relatively small. In our previous study, we reported results with 103 cases of RP using nested RT-PCR for detection of PSCA mRNA; our findings showed $P S C A$ mRNA positivity in the peripheral blood was related to BCR after RP in high-risk disease. Another report with nested RT-PCR using peripheral blood samples in locally advanced disease demonstrated similar results [17].

In the era of precision medicine, treatment of PC will be tailored based on the characteristics of PC in each patient. Liquid biopsy using circulating tumor cells is a promising tool for assessment of response to therapies and prognosis in patients with PC. RT-PCR has also been used for highly sensitive detection of circulating tumor cells. RT-PCR for analysis of circulating tumor cells enables follow-up of biopsy analysis and is minimally invasive. Thus, RT-PCR is a suitable tool for predicting prognosis or monitoring treatment response, and liquid biopsy using RT-PCR with enhanced accuracy is urgently required for personalized treatment of PC. The present study showed that PSCA mRNA positivity by RT-PCR may be a useful tool for predicting treatment outcomes in patients with PC.

The present study has some limitations. Since the study was conducted with data from a single institution, selection bias may have occurred. Second, since the RP was performed by two operators, the difference of the operator might influence the outcomes. Third, with regard to the survival outcome, there was limited number of death events due to limited follow-up duration. Despite of these limitations, we believe that the findings of the present study are highly relevant because this 
Table 2: Univariate and multivariate analyses of prognostic factors for biochemical recurrence

\begin{tabular}{lccc}
\hline Variables & HR & 95\% CI & $p$ value \\
\hline Univariate & & & 0.903 \\
Age & 1.002 & $0.969-1.036$ & $<0.001$ \\
PSA $\geq 20$ & 2.344 & $1.581-3.475$ & $<0.001$ \\
Biopsy GS $\geq 8$ & 2.626 & $1.718-4.012$ & 0.012 \\
RT-PCR PSCA positivity & 1.673 & $1.119-2.500$ & $<0.001$ \\
Pathologic stage $\geq$ T3 & 3.844 & $2.435-6.174$ & \\
Multivariate & & & 0.031 \\
Age & & & $<0.001$ \\
PSA $\geq 20$ & 1.576 & $1.043-2.382$ & $<0.001$ \\
Biopsy GS $\geq 8$ & 2.247 & $1.632-3.931$ & $<0.001$ \\
RT-PCR PSCA positivity & 1.817 & $1.504-3.192$ & $2.084-5.585$ \\
Pathologic stage $\geq$ T3 & 3.411 & & \\
\hline Abs & & \\
\hline
\end{tabular}

Abbreviations: PSA, prostate-specific antigen; GS, Gleason score; RT-PCR, reverse transcription polymerase chain reaction; PSCA, prostate stem cell antigen.

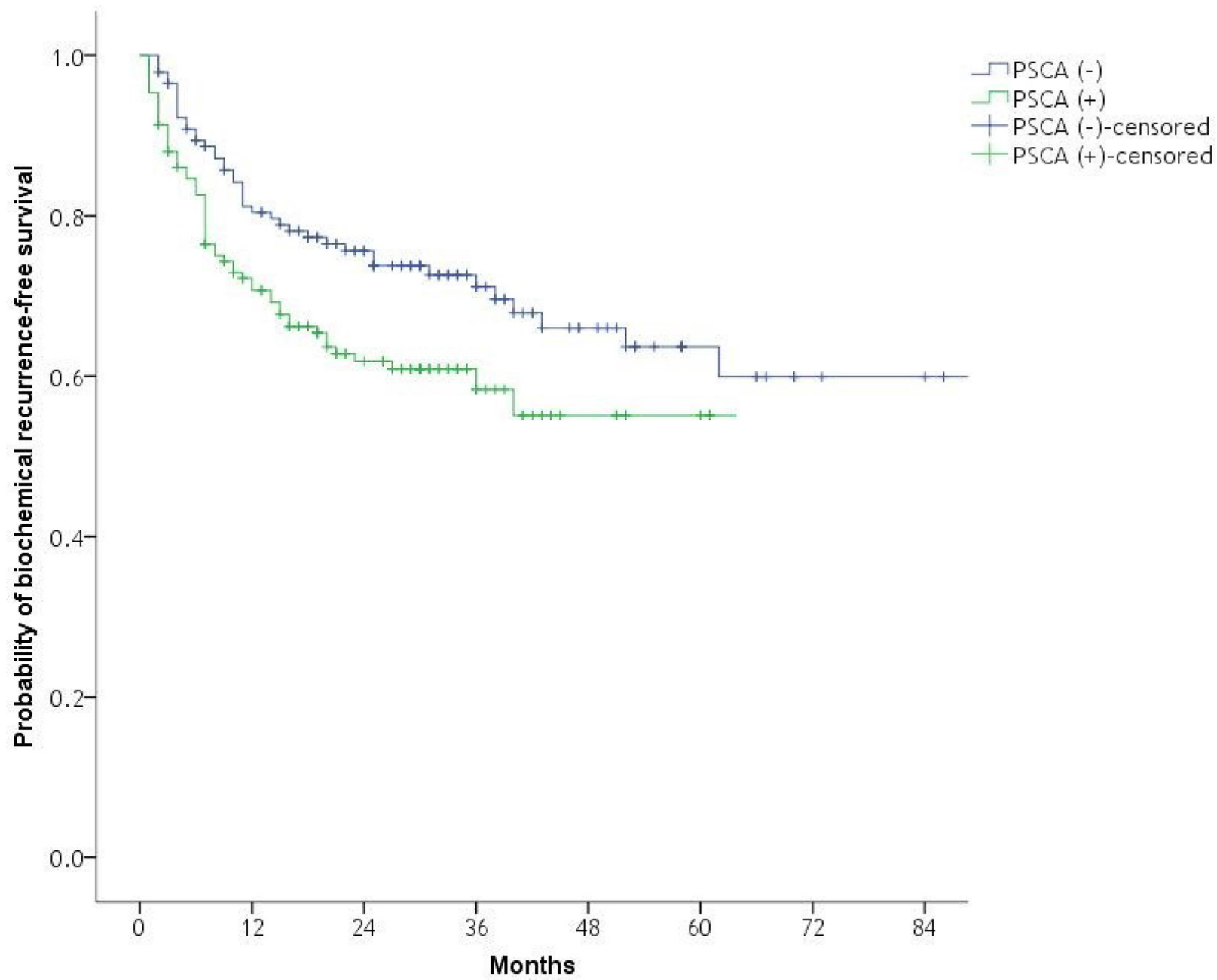

Figure 1: Kaplan-Meier plot of the likelihood of biochemical recurrence-free survival with respect to RT-PCR PSCA positivity after radical prostatectomy. Log-rank test, $p=0.010$. RT-PCR, reverse transcription polymerase chain reaction; PSCA, prostate stem cell antigen. 

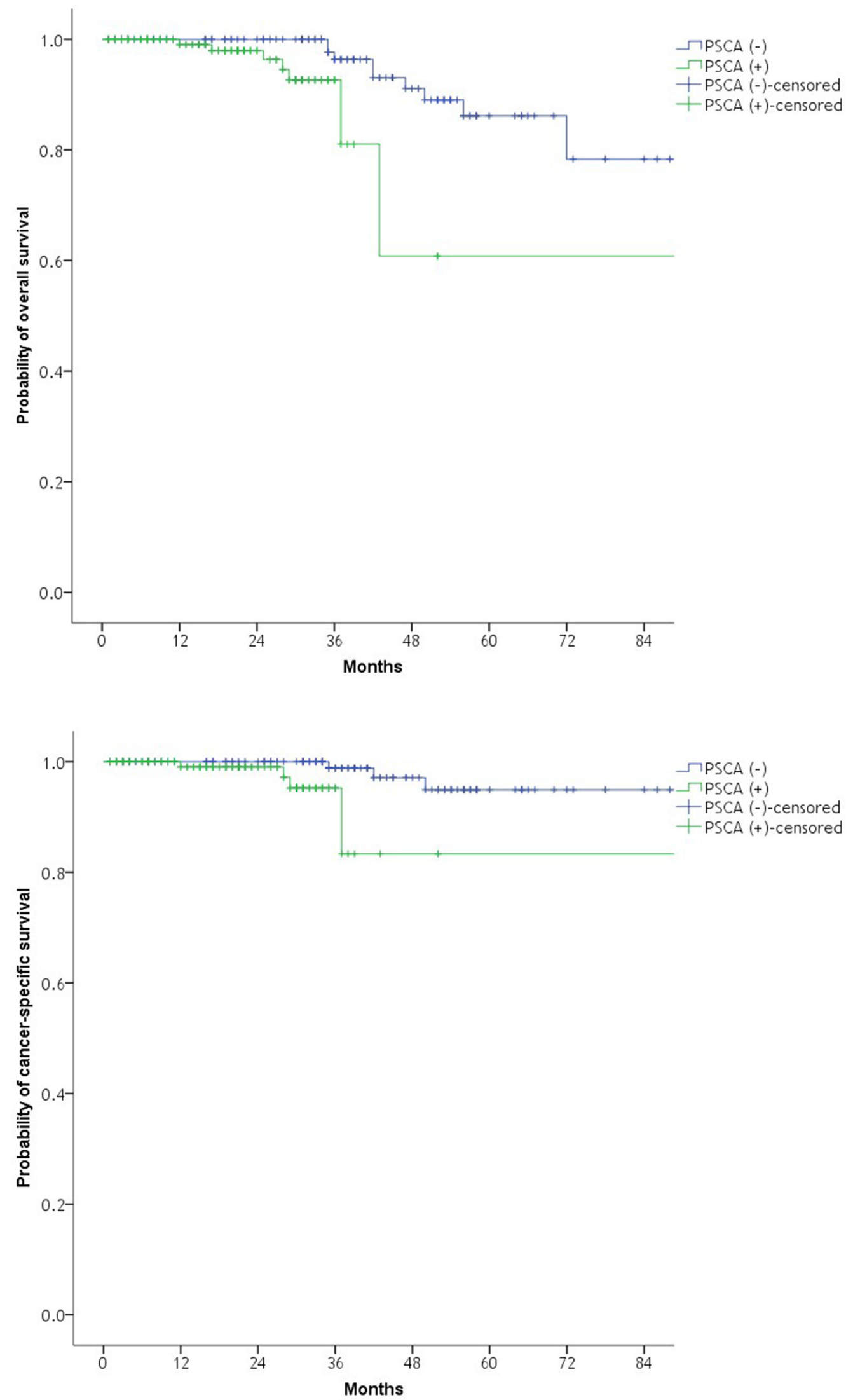

Figure 2: Kaplan-Meier plot of the likelihood of overall survival and cancer-specific survival with respect to RT-PCR PSCA positivity after radical prostatectomy. Log-rank test, $p=0.004$; Log-rank test, $p=0.014$. RT-PCR, reverse transcription polymerase chain reaction; PSCA, prostate stem cell antigen. 
Table 3: Univariate and multivariate analyses of prognostic factors of overall survival and cancer-specific survival

\begin{tabular}{|c|c|c|c|c|c|c|}
\hline \multirow[b]{2}{*}{ Variables } & \multicolumn{3}{|c|}{ OS } & \multicolumn{3}{|c|}{ CSS } \\
\hline & HR & $95 \% \mathrm{CI}$ & $p$ value & HR & $95 \% \mathrm{CI}$ & $p$ value \\
\hline \multicolumn{7}{|l|}{ Univariate } \\
\hline Age & 1.119 & $1.009-1.242$ & 0.034 & 1.060 & $0.915-1.227$ & 0.437 \\
\hline $\mathrm{PSA} \geq 20$ & 1.148 & $0.395-3.340$ & 0.800 & 2.972 & $0.665-13.284$ & 0.154 \\
\hline Biopsy GS $\geq 8$ & 4.253 & $1.398-12.940$ & 0.011 & 10.471 & $2.259-48.354$ & 0.003 \\
\hline RT-PCR PSCA positivity & 4.673 & $1.553-14.059$ & 0.006 & 6.702 & $1.284-34.975$ & 0.024 \\
\hline Pathologic stage $\geq \mathrm{T} 3$ & 2.597 & $1.020-8.571$ & 0.046 & 8.573 & $1.011-72.714$ & 0.049 \\
\hline \multicolumn{7}{|l|}{ Multivariate } \\
\hline \multicolumn{7}{|l|}{ Age } \\
\hline $\mathrm{PSA} \geq 20$ & 1.111 & $1.001-1.232$ & 0.048 & 3.120 & $0.462-16.108$ & 0.205 \\
\hline Biopsy GS $\geq 8$ & 3.514 & $1.113-11.214$ & 0.031 & 8.195 & $1.418-39.195$ & 0.012 \\
\hline RT-PCR PSCA positivity & 5.172 & $1.692-15.062$ & 0.005 & 12.784 & $1.799-96.582$ & 0.008 \\
\hline Pathologic stage $\geq \mathrm{T} 3$ & 1.910 & $0.674-5.182$ & 0.314 & 3.392 & $0.411-31.856$ & 0.274 \\
\hline
\end{tabular}

Abbreviations: PSA, prostate-specific antigen; GS, Gleason score; RT-PCR, reverse transcription polymerase chain reaction; PSCA, prostate stem cell antigen.

is the study with largest cohort evaluating the value of PSCA mRNA in peripheral blood as a marker for predicting BCR, OS, and CSS after RP in high-risk PC to date. In addition, homogenous composition of patients in regard to treatment modality is another strength of this study. In our previous study [11], patients who received neoadjuvant hormone therapy were included, hence the possibility that neoadjuvant hormone therapy influenced PSCA expression in peripheral blood could not be excluded.

The findings of the present study suggest that PSCA RT-PCR could provide urologists with useful information to identify and select patients with high-risk disease who are expected to show better oncologic outcome after RP. Additionally, our results provide insights into the optimal approach for selecting patients who require adjuvant therapies, such as adjuvant radiation therapy or hormone therapy.

In conclusion, the presence of PSCA mRNA in peripheral blood was related to poor prognosis with regard to BCR, OS, and CSS. Detection of PSCA mRNA by RTPCR in peripheral blood can be used to predict survival after RP in patients with high-risk PC. Future study with larger cohort and long-term follow-up is required to confirm this finding.

\section{MATERIALS AND METHODS}

\section{Ethics statement}

This study was approved by the Institutional Review Board (IRB) of our institution (IRB approval number: NCCNCS05049). All patient records were anonymized and de-identified before analysis. The study protocol was consistent with the ethical guidelines of the World Medical Association Declaration of Helsinki Ethical Principles for Medical Research Involving Human Subjects. Informed consent was obtained from all subjects for the collection of clinicopathological data, serum samples, and tissue samples.

\section{Subjects}

A total of 295 patients with high-risk PC who were scheduled to undergo RP were prospectively enrolled from 2008 to 2016 . Eligible patients had histologically proven prostate adenocarcinoma. High-risk PC was defined according to the presence of any one of the following criteria: clinical stage $\geq \mathrm{T} 2 \mathrm{c}$, serum PSA $\geq 20 \mathrm{ng} / \mathrm{mL}$, or biopsy GS $\geq 8$ [3]. All patients underwent RP and standard pelvic lymph node dissection. RP was performed by two urologists (KHL and JYJ).

\section{Blood samples, extraction of RNA, and reverse transcription}

Peripheral blood sampling was performed preoperatively. After Ficoll-Plaque plus gradient centrifugation (GE Healthcare, Little Chalfont, UK), nucleated cell fractions were separated from $5 \mathrm{~mL}$ whole blood samples that were anticoagulated with ethylenediaminetetraacetic acid (EDTA). Extraction of RNA and reverse transcription were conducted as performed in our prior report [18].

\section{PCR and nested PCR}

Primers were designed by Bioneer (Daejeon, Republic of Korea). The intron spanning primer pairs that were specific for human PSCA were as follows: sense, 
5'-TGCTTGCCCTGTTGATGGCAG-3', and antisense, 5'-ACGTGAGCCGGACGACGAGAC-3'. The antisense primer was replaced by 5'-TACTCCTGCAAAGC CCAGGT-3' for nested PCR. The housekeeping gene $\beta$-actin was used as an internal control. PCR was conducted for 25 cycles in a volume of $20 \mu \mathrm{L}$ containing $1 \mathrm{mM}$ sense and antisense primers, $1 \mathrm{~mL}$ RT product, $10 \mathrm{mM}$ Tris-HCL (pH 9.0), $1.5 \mathrm{mM} \mathrm{MgCl}, 0.25 \mathrm{mM}$ of each dNTP, $30 \mathrm{mM} \mathrm{KCl}$, and $1 \mathrm{U}$ Taq-DNA polymerase (Bioneer). For amplifying cDNA $(0.5 \mathrm{mg})$, a tubecontrolled thermal cycler (MJ Research, Waltham, MN, USA) was used. Amplification of PCR products $(2 \mathrm{~mL})$ was performed for additional 40 cycles utilizing the same reagents for nested PCR, although nested primers replaced the original primers.

\section{Sensitivity of RT-PCR assays and PCR product analysis}

The sensitivity of the RT-PCR assay was determined by measuring $\mathrm{LNCaP}$ human $\mathrm{PC}$ cell dilutions in peripheral blood mononuclear cell (PBMC) suspensions. RT-PCR assay sensitivity was determined as described in our previous study [18]. It was found that the mRNAs of $10 \mathrm{LNCaP}$ cells which was diluted in $10^{7}$ PBMCs from healthy donors could be detected by nested RT-PCR for PSCA. Samples that were subjected to qualitative RT-PCR for detection of PSCA mRNA were analyzed several times to confirm the reproducibility of the test. After the initial results were confirmed by re-assay, results for each sample were submitted for subsequent analyses. For PCR product analysis, a premix of $10 \mu \mathrm{L}$ PCR product with loading dye was evaluated using a PCR kit (Bioneer). Subsequently, samples were loaded onto agarose gels (2\%) in TBE buffer (0.1 M Tris [pH 8.4], $90 \mathrm{mM}$ boric acid, $1 \mathrm{mM}$ EDTA). The samples were then subjected to electrophoresis for 30 min. Staining of gels was performed using ethidium bromide, and visualization of amplicons was carried out with an ultraviolet transilluminator. To confirm internal consistency, scoring of assay results was performed by two investigators (WSP and MKC) who were blinded to any clinicopathological information.

\section{Pathological analysis}

Complete transverse sectioning was performed from the base to the apex of prostrate specimens with 4-mm intervals. Prostate specimens were examined by a single pathologist (WSP) and staged using the TNM system (International Union Against Cancer). GSs, the presence of ECE and SVI were evaluated.

\section{Follow-up}

All 295 patients were scheduled to undergo serum PSA evaluation with routine blood tests and routine checkups that included history taking and physical examination, such as digital rectal examination, every 3 months for the first 2 years after RP, biennially from the third to the fifth years, and then annually thereafter. Imaging evaluations using computed tomography scanning, magnetic resonance imaging, and bone scanning were evaluated if clinically indicated. BCR was defined as a sustained elevation of PSA more than $0.4 \mathrm{ng} / \mathrm{mL}$ on at least two occasions. Clinical outcome analyses were performed using the NCC PC database.

\section{Statistical analysis}

Independent-sample $t$-tests were used for betweengroup comparisons. Fisher's exact test was used to compare categorical data. The Kaplan-Meier method was used to evaluate survival outcomes, and the log-rank test was used to assess differences. Univariate and multivariate analyses were conducted with the Cox proportional hazards regression model. For deriving a final model of variables with independent significant relationships with survival, variables with $p$ values of less than 0.20 in univariate analysis were included in multivariate analysis. All statistical analyses were performed using SPSS software for Windows (version 20; SPSS, Chicago, IL, USA). A $p$ value of less than 0.05 was considered statistically significant, and all statistical tests were two-sided.

\section{Abbreviations}

PSCA: prostate stem cell antigen; PC: prostate cancer; BCR: biochemical recurrence; RP: radical prostatectomy; RT-PCR: reverse transcription polymerase chain reaction; HR: hazard ratio; CI: confidence interval; OS: overall survival; CSS: cancer-specific survival; IQR: interquartile range; PSA: prostate-specific antigen; GS: Gleason score; ECE: extracapsular extension; SVI: seminal vesicle invasion.

\section{Author contributions}

Study concept and design: Yoon Seok Suh, Jae Young Joung, Kang Hyun Lee. Acquisition of data: Yoon Seok Suh, Jae Young Joung, Jeong Eun Kim, Moon Kyung Choi. Analysis and interpretation of data: Yoon Seok Suh, Jae Young Joung, Sung Han Kim. Drafting of the manuscript: Yoon Seok Suh, Jae Young Joung. Critical revision of the manuscript for important intellectual content: Weon Seo Park, Sang-Jin Lee, Ho Kyung Seo, Jinsoo Chung, Kang Hyun Lee. Statistical analysis: Yoon Seok Suh, Sung Han Kim. Obtaining funding: Kang Hyun Lee. Administrative, technical, or material support: Jae Young Joung, Jeong Eun Kim, Moon Kyung Choi, Weon Seo Park, Sang-Jin Lee, Ho Kyung Seo, Jinsoo Chung, Kang Hyun Lee. Supervision: Weon Seo Park, Sang-Jin Lee, Kang Hyun Lee. Other (specify): none. 


\section{CONFLICTS OF INTEREST}

None declared.

\section{FUNDING}

This study was supported by a National Cancer Center Grant (no. 1510170-3).

\section{REFERENCES}

1. Torre LA, Bray F, Siegel RL, Ferlay J, Lortet-Tieulent J, Jemal A. Global cancer statistics, 2012. CA Cancer J Clin. 2015; 65:87-108.

2. Siegel RL, Miller KD, Jemal A. Cancer statistics, 2016. CA Cancer J Clin. 2016; 66:7-30.

3. D'Amico AV, Whittington R, Malkowicz SB, Schultz D, Blank K, Broderick GA, Tomaszewski JE, Renshaw AA, Kaplan I, Beard CJ, Wein A. Biochemical outcome after radical prostatectomy, external beam radiation therapy, or interstitial radiation therapy for clinically localized prostate cancer. JAMA. 1998; 280:969-74.

4. Marciscano AE, Hardee ME, Sanfilippo N. Management of high-risk localized prostate cancer. Adv Urol. 2011; 2012.

5. Bolla M, Gonzalez D, Warde P, Dubois JB, Mirimanoff RO, Storme G, Bernier J, Kuten A, Sternberg C, Gil T, Collette L, Pierart M. Improved survival in patients with locally advanced prostate cancer treated with radiotherapy and goserelin. N Engl J Med. 1997; 337:295-300.

6. Bolla M, Collette L, Blank L, Warde P, Dubois JB, Mirimanoff RO, Storme G, Bernier J, Kuten A, Sternberg C, Mattelaer J, Lopez Torecilla J, Pfeffer JR, et al. Long-term results with immediate androgen suppression and external irradiation in patients with locally advanced prostate cancer (an EORTC study): a phase III randomised trial. Lancet. 2002; 360:103-06.

7. Bolla M, Van Tienhoven G, Warde $P$, Dubois JB, Mirimanoff RO, Storme G, Bernier J, Kuten A, Sternberg C, Billiet I, Torecilla JL, Pfeffer R, Cutajar CL, et al. External irradiation with or without long-term androgen suppression for prostate cancer with high metastatic risk: 10-year results of an EORTC randomised study. Lancet Oncol. 2010; 11:1066-73.

8. Ischia J, Gleave M. Radical prostatectomy in high-risk prostate cancer. Int J Urol. 2013; 20:290-300.
9. Yossepowitch O, Eastham JA. Radical prostatectomy for high-risk prostate cancer. World J Urol. 2008; 26:219-24.

10. Jenkins RB, Qian J, Lieber MM, Bostwick DG. Detection of c-myc oncogene amplification and chromosomal anomalies in metastatic prostatic carcinoma by fluorescence in situ hybridization. Cancer Res. 1997; 57:524-31.

11. Joung JY, Cho KS, Kim JE, Seo HK, Chung J, Park WS, Choi MK, Lee KH. Prostate stem cell antigen mRNA in peripheral blood as a potential predictor of biochemical recurrence in high-risk prostate cancer. J Surg Oncol. 2010; 101:145-48.

12. Yossepowitch O, Eggener SE, Serio AM, Carver BS, Bianco FJ Jr, Scardino PT, Eastham JA. Secondary therapy, metastatic progression, and cancer-specific mortality in men with clinically high-risk prostate cancer treated with radical prostatectomy. Eur Urol. 2008; 53:950-59.

13. Yossepowitch O, Eggener SE, Bianco FJ Jr, Carver BS, Serio A, Scardino PT, Eastham JA. Radical prostatectomy for clinically localized, high risk prostate cancer: critical analysis of risk assessment methods. J Urol. 2007; 178:493-99.

14. Reiter RE, Gu Z, Watabe T, Thomas G, Szigeti K, Davis E, Wahl M, Nisitani S, Yamashiro J, Le Beau MM, Loda M, Witte ON. Prostate stem cell antigen: a cell surface marker overexpressed in prostate cancer. Proc Natl Acad Sci USA. 1998; 95:1735-40.

15. Oefelein MG, Ignatoff JM, Clemens JQ, Watkin W, Kaul KL. Clinical and molecular followup after radical retropubic prostatectomy. J Urol. 1999; 162:307-10.

16. Zippelius A, Pantel K. RT-PCR-based detection of occult disseminated tumor cells in peripheral blood and bone marrow of patients with solid tumors. An overview. Ann N Y Acad Sci. 2000; 906:110-23.

17. Okegawa T, Noda H, Kato M, Miyata A, Nutahara K, Higashihara E. Value of reverse transcription polymerase chain reaction assay in pathological stage $\mathrm{T} 3 \mathrm{~N} 0$ prostate cancer. Prostate. 2000; 44:210-18.

18. Joung JY, Yang SO, Jeong IG, Han KS, Seo HK, Chung J, Park WS, Lee KH. Reverse transcriptase-polymerase chain reaction and immunohistochemical studies for detection of prostate stem cell antigen expression in prostate cancer: potential value in molecular staging of prostate cancer. Int $\mathbf{J}$ Urol. 2007; 14:635-43. 